\title{
Dynamics of Interacting Tachyonic Teleparallel Dark Energy
}

\begin{abstract}
Ali Banijamali
Department of Basic Sciences, Babol University of Technology, Babol 47148-71167, Iran

Correspondence should be addressed to Ali Banijamali; a.banijamali@nit.ac.ir

Received 10 February 2014; Revised 6 May 2014; Accepted 7 May 2014; Published 3 June 2014

Academic Editor: George Siopsis

Copyright (C) 2014 Ali Banijamali. This is an open access article distributed under the Creative Commons Attribution License, which permits unrestricted use, distribution, and reproduction in any medium, provided the original work is properly cited. The publication of this article was funded by SCOAP ${ }^{3}$.

We consider a tachyon scalar field which is nonminimally coupled to gravity in the framework of teleparallel gravity. We analyze the phase-space of the model, known as tachyonic teleparallel dark energy, in the presence of an interaction between dark energy and background matter. We find that although there exist some late-time accelerated attractor solutions, there is no scaling attractor. So, unfortunately interacting tachyonic teleparallel dark energy cannot alleviate the coincidence problem.
\end{abstract}

\section{Introduction}

Recent astrophysical observations including Supernova Ia [1, $2]$, large scale structure $[3,4]$, the baryon acoustic oscillations [5], and cosmic microwave background radiation [6-9] have indicated that our universe is experiencing an accelerating phase of expansion.

There are two ways to explain the current cosmic expansion. The first one is to add extra terms in the gravitational Lagrangian or modify gravity on large scales and the second one is to introduce an unknown energy component, dubbed as dark energy with negative pressure.

The simplest modification of general relativity is the socalled $f(R)$ gravity in which one generalizes the EinsteinHilbert action to a general function of the Ricci scalar $R$ (for reviews see [10-13]). In the other side the simplest candidate of dark energy is the vacuum energy or the cosmological constant $\Lambda$ with a constant equation of state parameter $\omega=-1$. However, the cosmological constant suffers from serious problems such as huge amount of fine tuning required for its magnitude and lack of dynamics [14-17]. Due to these problems numerous dynamical dark energy models have been proposed. Quintessence [18-22], phantom [23-29], tachyon [30,31], and the combination of quintessence and phantom in a unified model named quintom [32-38] are wellknown examples of dark energy models (for reviews on dark energy models, see $[39,40])$.
Although usually the scalar fields are minimally coupled to gravity, there are compelling reasons (e.g., quantum corrections and renormalizability of the scalar field theory in curved space) to include an explicit nonminimal coupling between dark energy and gravity in the action [41-48]. Also, a possible coupling between dark energy and dark matter can be included in generalized versions of the aforementioned models $[49,50]$. Because of this possibility, various forms of interacting dark energy models have been constructed hitherto [51-65].

Furthermore, an equivalent form of classical gravity is the so-called teleparallel gravity, in which, instead of using the torsionless Levi-Civita connection, one uses the curvatureless Weitzenbock one. In this theory, the dynamical variables are a set of four tetrad (or vierbein) fields that form the pseudoorthogonal bases for the tangent space at each point of spacetime [66-68]. The teleparallel Lagrangian density $T$ can be constructed from torsion tensor and only differs with the Ricci scalar by a total divergence. Thus, apart from some conceptual differences, general relativity and teleparallel gravity are dynamically equivalent theories and indistinguishable from general relativity at the level of field equations $[67,68]$. Recently, $f(R)$ inspired teleparallel gravity, the so-called $f(T)$ gravity, has attracted much attention [69-83].

Teleparallel dark energy is a recently proposed scenario in which a nonminimal coupling between quintessence and gravity in the framework of teleparallel gravity was considered $[84,85]$. This theory has a rich structure and its dynamics 
was studied in [86-88]. Tachyonic teleparallel dark energy is a generalization of teleparallel dark energy by inserting a noncanonical scalar field instead of quintessence in the action [89]. Phase-space analysis of this model has been investigated in [90]. In this paper we study the dynamics of interacting tachyonic teleparallel dark energy. We find stable solutions of the model which are late-time accelerated attractors and correspond to dark energy dominated solutions. Thus, coincidence problem cannot be solved in interacting tachyonic teleparallel dark energy model.

An outline of the present letter is as follows. In Section 2 we briefly review teleparallel gravity. In Section 3 we present tachyonic teleparallel dark energy and obtain energy density, pressure, and equation of state of the model. In Section 4 we study dynamics of the model in a system of autonomous differential equations, find its fixed points, and study their stabilities. Section 5 is devoted to conclusions.

\section{Teleparallel Gravity}

Let us start with a brief review of the key ingredients of teleparallel gravity [66-72]. In this theory, the metric tensor is obtained from the dual vierbein as

$$
g_{\mu \nu}=\eta_{i j} e_{\mu}^{i} e_{\nu}^{j}
$$

where Latin $i, j$ are indices running over $0,1,2,3$ for the tangent space at each point $x^{\mu}$ of the manifold and Greek $\mu$ and $\nu$ are coordinate indices on the manifold, taking the values $0,1,2,3$. Furthermore, the torsion tensor of the Weitzenbock connection $\Gamma_{\nu \mu}^{\rho}[91]$ reads

$$
T_{\mu \nu}^{\rho}=\Gamma_{\nu \mu}^{\rho}-\Gamma_{\mu \nu}^{\rho}=e_{i}^{\rho}\left(\partial_{\mu} e_{\nu}^{i}-\partial_{\nu} e_{\mu}^{i}\right)
$$

In the present formalism all the information concerning the gravitational field is included in the torsion tensor $T_{\mu \nu}^{\rho}$. The corresponding teleparallel Lagrangian can be constructed from this torsion tensor under the assumptions of invariance under general coordinate transformation, global Lorentz transformations, and the parity operation, along with requiring the Lagrangian density to be second order in the torsion tensor [92]. The starting action in a universe governed by teleparallel gravity is

$$
S=\int d^{4} x e\left[\frac{T}{2 \kappa^{2}}+\mathscr{L}_{m}\right]
$$

where $e=\operatorname{det}\left(e_{\mu}^{i}\right)=\sqrt{-g}$ and $T$ is the torsion scalar given by

$$
\mathscr{L}=T=\frac{1}{4} T^{\rho \mu \nu} T_{\rho \mu \nu}+\frac{1}{2} T^{\rho \mu \nu} T_{\nu \mu \rho}-T_{\rho \mu}^{\rho} T_{\nu}^{\nu \mu} .
$$

Variation with respect to the vierbein fields yields to the equations of motion which are exactly the same as those of general relativity for every geometry choice. Since the action (3) in a Friedmann-Robertson-Walker (FRW) background is equivalent to a matter domination universe in the framework of general relativity, hence it cannot be accelerated. So, we should generalize action (3) either by replacing $T$ with $f(T)$
[69-83] or by adding a scalar field responsible for dark energy in teleparallel gravity. On the other hand, one can easily find that dark energy in the framework of teleparallel gravity is completely identical to the one in the framework of general relativity, and hence there is nothing new. Recently Geng et al. [84] have proposed to modify action (3) by including a nonminimal coupling between quintessence and gravity in the framework of teleparallel gravity and named it teleparallel dark energy. Here we generalize teleparallel dark energy model by replacing canonical scalar field (quintessence) by a noncanonical scalar field. The noncanonical scalar field is tachyon field and the model has been called tachyonic teleparallel dark energy.

\section{Interacting Tachyonic Teleparallel Dark Energy}

The action of teleparallel dark energy with a nonminimal coupling between tachyon field and teleparallel gravity reads

$$
S=\int d^{4} x e\left[\frac{T}{2 \kappa^{2}}+\xi f(\varphi) T-V(\varphi) \sqrt{1-2 X}+\mathscr{L}_{m}\right],
$$

where $\xi$ is a dimensionless constant and $f(\varphi)$ is an arbitrary function of scalar field and it is responsible for nonminimal coupling between dark energy and gravity. $V(\varphi)$ is the tachyonic potential and $X=(1 / 2) \partial_{\mu} \varphi \partial^{\mu} \varphi$ (we use the metric signature $(+,-,-,-))$. We should emphasize that in torsion formulation of general relativity the only scalar is the torsion scalar and hence the nonminimal coupling will be between $T$ and tachyon field in analogy with the standard nonminimal tachyon cosmology in general relativity where the scalar field couples to the Ricci scalar.

Phase-space analysis of tachyon field in the framework of general relativity has been studied in several papers (see, e.g., [93-95]). To obtain a closed autonomous system of ordinary differential equations out of the cosmological field equations [96] has proposed the following transformation of the tachyon field:

$$
\varphi \longrightarrow \phi=\int d \varphi \sqrt{V(\varphi)} \Longleftrightarrow \partial \varphi=\frac{\partial \phi}{\sqrt{V(\phi)}} .
$$

It is shown that the preceding transformation can help us study tachyon dynamics for a wide class of self-interaction potentials beyond the inverse square one. It is revealed that for power law potentials the late-time attractor is always the de Sitter solution while for sinh-like potentials the late-time attractor can be either the inflationary tachyon dominated solution or the matter scaling phase.

In this paper we also use the field redefinition (6) to study the dynamics of the interacting tachyon field nonminimally coupled with gravity in the framework of teleparallel gravity. Therefore, our starting action is obtained using (6) in (5):

$$
S=\int d^{4} x e\left[\frac{T}{2 \kappa^{2}}+\xi f(\phi) T-V(\phi) \sqrt{1-\frac{2 X}{V(\phi)}}+\mathscr{L}_{m}\right] .
$$


Also we consider a possible interaction between dark energy and dark matter because there is no physical argument to exclude the interaction between them.

In a spatially flat FRW space-time,

$$
d s^{2}=d t^{2}-a^{2}(t)\left(d r^{2}+r^{2} d \Omega^{2}\right),
$$

and a vierbein choice of the form $e_{\mu}^{i}=\operatorname{diag}(1, a, a, a)$, the equation of motion of the scalar field can be obtained by variation of the action (7) with respect to $\phi$ :

$$
\ddot{\phi}+3 \mu^{-2} H \dot{\phi}+\left(1-\frac{3 X}{V(\phi)}\right) V_{, \phi}+6 \xi \mu^{-3} H^{2} f_{, \phi}=-\frac{Q}{\dot{\phi}},
$$

with $Q$ being a general interaction coupling term between dark energy and dark matter. Furthermore, the effective energy density and pressure of tachyonic dark energy read

$$
\begin{aligned}
& \rho_{\phi}=\mu V(\phi)-6 \xi H^{2} f(\phi), \\
& P_{\phi}=-\mu^{-1} V(\phi)+2 \xi\left(3 H^{2}+2 \dot{H}\right) f(\phi)+4 \xi H f_{, \phi} \dot{\phi},
\end{aligned}
$$

where $f_{, \phi}=d f / d \phi$ and $\mu=1 / \sqrt{1-2 X / V}$.

In (9) and (10) we have used the useful relation,

$$
T=-6 H^{2}
$$

which simply arises from the calculation of (4) for the FRW metric (8). The scalar field evolution (9) expresses the continuity equation for the field and matter as follows:

$$
\begin{aligned}
& \dot{\rho}_{\phi}+3 H\left(1+\omega_{\phi}\right) \rho_{\phi}=-Q, \\
& \dot{\rho}_{m}+3 H\left(1+\omega_{m}\right) \rho_{m}=Q
\end{aligned}
$$

where $\omega_{\phi}=P_{\phi} / \rho_{\phi}$ is the equation of state parameter of dark energy which is attributed to the scalar field $\phi$. The barotropic index is defined by $\gamma \equiv 1+\omega_{m}$ with $0<\gamma<2$.

In FRW background (8) the corresponding Friedmann equations are given by

$$
\begin{gathered}
H^{2}=\frac{\kappa^{2}}{3}\left(\rho_{\phi}+\rho_{m}\right), \\
\dot{H}=-\frac{\kappa^{2}}{2}\left(\rho_{\phi}+P_{\phi}+\rho_{m}+P_{m}\right),
\end{gathered}
$$

where $H=\dot{a} / a$ is the Hubble parameter and a dot stands for the derivative with respect to the cosmic time $t$. In these equations, $\rho_{m}$ and $P_{m}$ are the matter energy density and the pressure, respectively.

Tachyonic teleparallel dark energy has been studied in [89]. It is shown that such a scenario can realize phantom divide crossing during its evolution and so it exhibits very interesting cosmological behavior. Therefore, it seems necessary to perform a phase-space analysis of such theory. In phase-space analysis we investigate late-time solutions that are independent from the initial conditions and the specific universe evolution.

\section{Phase-Space Analysis}

In order to study the phase-space and stability of the model (7), we should translate the evolution equations in the language of the autonomous dynamical system $X^{\prime}=f(X)$ [97$100]$, where $X$ is the column vector constituted by suitable auxiliary variables, $f(X)$ is the corresponding column vector of the autonomous equations, and prime denotes derivative with respect to the logarithm of the scale factor $N=\ln a$. The critical points $X_{c}$ are extracted from $X^{\prime}=0$ and in order to determine the stability properties of these critical points we expand around $X_{c}$, setting $X=X_{c}+U$ with $U$ the perturbations of the variables considered as column vector. Thus, up to the first order we acquire $U^{\prime}=M \cdot U$, where the matrix $M$ contains the coefficients of the perturbation equations. For each critical point, the eigenvalues of $M$ determine its type and stability.

Now, let us transform the cosmological equations into an autonomous dynamical system. To this end following, for example, [101-106] we introduce the following auxiliary variables:

$$
x \equiv \frac{\dot{\phi}}{\sqrt{V}}, \quad y \equiv \frac{\kappa \sqrt{V}}{\sqrt{3} H}, \quad u \equiv \kappa \sqrt{f} .
$$

The auxiliary variables allow us to straightforwardly obtain the density parameter of dark energy and dark matter:

$$
\begin{gathered}
\Omega_{\mathrm{DE}} \equiv \frac{\kappa^{2} \rho_{\mathrm{DE}}}{3 H^{2}}=\mu y^{2}-2 \xi u^{2}, \\
\Omega_{m} \equiv \frac{\kappa^{2} \rho_{m}}{3 H^{2}}=1-\Omega_{\mathrm{DE}}
\end{gathered}
$$

while the equation of state of the field reads

$$
\begin{aligned}
\omega_{\mathrm{DE}} & \equiv \omega_{\phi}=\frac{P_{\phi}}{\rho_{\phi}} \\
& =\frac{-\mu^{-1} y^{2}+2 \xi u[(2 \sqrt{3} / 3) \alpha x y+u(1-(2 / 3) s)]}{\mu y^{2}-2 \xi u^{2}},
\end{aligned}
$$

where $\alpha \equiv f_{, \phi} / \sqrt{f}$ and

$$
s=-\frac{\dot{H}}{H^{2}}=\frac{4 \sqrt{3} \alpha \xi u x y+3 \mu\left(x^{2}-\gamma\right) y^{2}}{2\left(2 \xi u^{2}+1\right)}+\frac{3 \gamma}{2} .
$$

Other quantities with great physical significance, namely, the total equation of state parameter and the deceleration parameter, are given by

$$
\begin{aligned}
\omega_{\text {tot }} & \equiv \frac{P_{\phi}+P_{m}}{\rho_{\phi}+\rho_{m}} \\
& =\mu y^{2}\left(x^{2}-\gamma\right)+2 \xi u\left[\frac{2 \sqrt{3}}{3} \alpha x y+u\left(\gamma-\frac{2}{3} s\right)\right]+\gamma-1,
\end{aligned}
$$


TABLE 1: Location and existence conditions of the critical points and the corresponding values of the dark energy equation of state parameter $\omega_{\mathrm{DE}}$, of the total equation of state parameter $\omega_{\mathrm{tot}}$, and of the deceleration parameter $q$.

\begin{tabular}{|c|c|c|c|c|c|}
\hline Label & Location of $\left(x_{c}, y_{c}, u_{c}\right)$ & $\omega_{\mathrm{DE}}$ & $\omega_{\text {tot }}$ & $q$ & Existence \\
\hline$A$ & $0,1,0$ & -1 & -1 & -1 & $\lambda=0$ \\
\hline$B$ & $0,-1,0$ & -1 & -1 & -1 & $\lambda=0$ \\
\hline C & $0, \sqrt{\frac{\alpha}{\lambda^{2}}\left(\alpha \xi+\sqrt{\xi\left(\alpha^{2} \xi-2 \lambda^{2}\right)}\right)}, \frac{\alpha \xi+\sqrt{\xi\left(\alpha^{2} \xi-2 \lambda^{2}\right)}}{2 \xi \lambda}$ & -1 & -1 & -1 & $\begin{array}{c}\xi \geq \frac{2 \lambda^{2}}{\alpha^{2}} \text { and } \frac{\lambda}{\alpha}>0 \\
\quad \text { or } \\
\xi<0, \quad \alpha>0 \text { and } \lambda<0\end{array}$ \\
\hline$D$ & $0, \sqrt{\frac{\alpha}{\lambda^{2}}\left(\alpha \xi-\sqrt{\xi\left(\alpha^{2} \xi-2 \lambda^{2}\right)}\right)}, \frac{\alpha \xi-\sqrt{\xi\left(\alpha^{2} \xi-2 \lambda^{2}\right)}}{2 \xi \lambda}$ & -1 & -1 & -1 & $\begin{array}{c}\xi \geq \frac{2 \lambda^{2}}{\alpha^{2}} \text { and } \frac{\lambda}{\alpha}>0 \\
\quad \text { or } \\
\xi<0, \quad \alpha<0 \text { and } \lambda>0\end{array}$ \\
\hline
\end{tabular}

$$
\begin{aligned}
q \equiv & -1-\frac{\dot{H}}{H^{2}}=\frac{1}{2}+\frac{3}{2} \omega_{\text {tot }} \\
= & \frac{3}{2} \mu y^{2}\left(x^{2}-\gamma\right)+\xi u[2 \sqrt{3} \alpha x y+u(3 \gamma-2 s)] \\
& +\frac{3 \gamma}{2}-1
\end{aligned}
$$

We mention that relations (19) are always valid, that is, independently of the specific state of the system (they are valid in the whole phase-space and not only at the critical points).

With the help of the auxiliary variables (14), the equations of motion (9) and (13) can be rewritten as a dynamical system; namely,

$$
\begin{aligned}
x^{\prime}= & \frac{\sqrt{3}}{2}\left[\lambda x^{2} y+\lambda\left(2-3 x^{2}\right) y-4 \alpha \xi u \mu^{-3} y^{-1}-2 \sqrt{3} x \mu^{-2}\right] \\
& -\widehat{Q} \\
y^{\prime}= & \left(-\frac{\sqrt{3}}{2} \lambda x y+s\right) y, \\
u^{\prime}= & \frac{\sqrt{3} \alpha x y}{2},
\end{aligned}
$$

where $\widehat{Q}=Q / \dot{\phi} H \sqrt{V(\phi)}$ and $\lambda \equiv-V_{, \phi} / \kappa V$. Equations (20) can be an autonomous system when interaction term $Q$ is chosen to be a suitable form. From now on we assume the nonminimal coupling function to be $f(\phi) \propto \phi^{2}$ such that $\alpha$ is a constant. On the other hand the usual assumption in the literatures is to consider an exponential potential of the form $V=V_{0} e^{-k \lambda \phi}[107-110]$. Such a potential leads a constant $\lambda$. In fact $\lambda=-V_{, \phi} / k V \simeq$ const is valid for arbitrary but nearly flat potentials $[111,112]$. Note that an exponential potential of the form $V=V_{0} e^{-k \lambda \phi}$ is equivalent to the inverse square potential $V(\phi) \propto \phi^{-2}$ in terms of untransformed field $\phi$. Moreover, since the densities of dark energy and dark matter are nearly equal today there may be some coupling or interaction between them. Thus, various forms of interacting dark energy models [113-115] have been constructed in order to fulfil the observational requirements. In these models different forms of the coupling between dark energy and dark matter were proposed. Here we consider the most familiar interaction term extensively considered in the literature $Q=\beta \kappa \rho_{m} \dot{\phi}$ where $\beta$ is a constant $[107,108,116]$. Note that an interaction between dark matter and dark energy is a reasonable assumption but there is no reason to consider that baryonic matter is also coupled.

Using the above mentioned points we have a threedimensional autonomous system as follows:

$$
\begin{aligned}
x^{\prime}= & \frac{\sqrt{3}}{2}\left[\lambda x^{2} y+\lambda\left(2-3 x^{2}\right) y-4 \alpha \xi u \mu^{-3} y^{-1}-2 \sqrt{3} x \mu^{-2}\right] \\
& -\sqrt{3} \beta y^{-1} \Omega_{m}, \\
y^{\prime}= & \left(-\frac{\sqrt{3}}{2} \lambda x y+s\right) y, \\
u^{\prime}= & \frac{\sqrt{3} \alpha x y}{2} .
\end{aligned}
$$

Now, let us proceed to the phase-space analysis. The critical or fixed points $\left(x_{c}, y_{c}, u_{c}\right)$ of the autonomous system (21) are obtained by setting the left hand sides of the equations to zero, namely, by imposing the conditions $x^{\prime}=y^{\prime}=u^{\prime}=0$. One should note that we are interested in solutions which imply expanding solution, nonnegative dimensionless energy density parameters for the different species.

After some algebraic calculus, we find four critical points $(A, B, C, D)$ presented in Table 1 . In the same table we have provided the existence conditions of each point and the values of $\omega_{\mathrm{DE}}, \omega_{\text {tot }}$, and $q$ which can be used to discuss whether there exists acceleration phase or not. To study the stability of the critical points we substitute linear perturbations $x \rightarrow$ $x_{c}+\delta x, y \rightarrow y_{c}+\delta y$, and $u \rightarrow u_{c}+\delta u$ about the critical point $\left(x_{c}, y_{c}, u_{c}\right)$ into the autonomous system (21). The 
TABLE 2: The eigenvalues of the linearization matrices corresponding to the critical points. Here $B^{+}=\left(\alpha \xi+\sqrt{\xi\left(\alpha^{2} \xi-2 \lambda^{2}\right)}\right)$ and $B^{-}=$ $\left(\alpha \xi-\sqrt{\xi\left(\alpha^{2} \xi-2 \lambda^{2}\right)}\right)$.

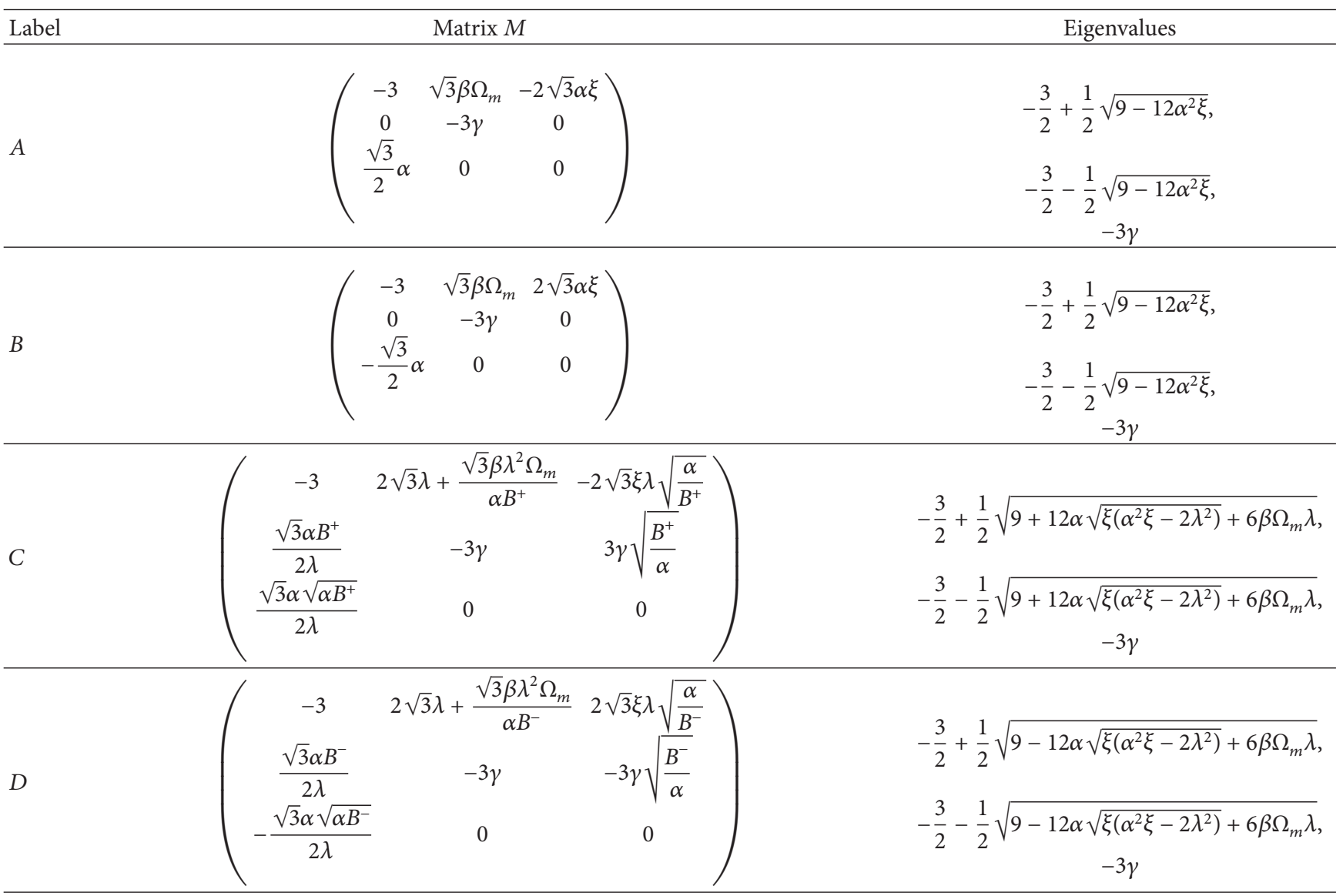

$3 \times 3$ matrix $M$ of the linearized perturbation equations of the autonomous system is shown in the appendix. Therefore, for each critical point of Table 1 we examine the sign of the real part of the eigenvalues of $M$, namely, $r_{1}, r_{2}$, and $r_{3}$, to determine the type and stability of the critical point. A fixed point is unstable if $r_{1}>0, r_{2}>0$, and $r_{3}>0$. It is saddle if the real part of the eigenvalues has different signs and it is stable for negative real part of eigenvalues. In Table 2 we have calculated the linearized perturbation matrix at each fixed point and corresponding eigenvalues. The stability conditions of each point are presented in Table 3.

We are going now to discuss the corresponding cosmological behavior of each critical point.

Critical Point $A$. Point $A$ represents a dark energy dominated solution that exists for all values of $\alpha$ and $\beta$. For $0<\xi<3 \alpha^{2} / 4$ it is a stable point (meaning that if the universe reaches this solution, it remains there forever) and thus it can attract the universe at late time. Accelerated expansion occurs for this point because $\omega_{\text {tot }}<-1 / 3$. Dark energy equation of state at this point is the same as the equation of state of the cosmological constant $\omega_{\mathrm{DE}}=-1$. Point $A$ has the disadvantage that exists for the limiting case $\lambda=0$ that is for a constant potential. Figure 1 from left to right shows the projections of the phase-space trajectories on the $y-x, u-y$, and $x-u$ planes for $\xi=0.5$ and $\alpha=1.5$. With these values of the parameters point $A$ is an attractor as it is clear in Figure 1. Also, we can see this stable point in 3-dimensional figure plotted in Figure 4. Also, point $A$ could be a saddle point (meaning that the universe during its evolution can reach this state but does not remain there and evolves to another state) for negative values of the coupling parameter $\xi$.

Critical Point $B$. The critical point $B$ also exists independent of the values of the $\alpha$ and $\beta$ but again for a constant potential. It corresponds to a completely dark energy dominated solution and could be a stable point if $0<\xi<3 \alpha^{2} / 4$. Note however that since the variable $y$ has the sign of $H$ thus this critical point corresponds to a contracting universe and it is not a physically meaningful solution.

Critical Point $C$. Accelerating dark energy dominated solution $C$ exists for $\xi \geq 2 \lambda^{2} / \alpha^{2}$ and $\lambda / \alpha>0$ or $\xi<0, \alpha>0$ and $\lambda<0$. This point is an attractor solution if there are the following constraints on the nonminimal coupling parameters:

$$
\begin{aligned}
& \frac{\lambda^{2}}{\alpha^{2}}\left(1+\sqrt{1+\frac{\beta^{2} \Omega_{m}^{2}}{4 \lambda^{2}}}\right) \\
& \quad<\xi<\frac{\lambda^{2}}{\alpha^{2}}\left(1+\sqrt{1+\frac{9\left(1+(2 / 3) \beta \Omega_{m} \lambda\right)^{2}}{16 \lambda^{4}}}\right)
\end{aligned}
$$


TABLE 3: Stability of the critical points of Table 1.

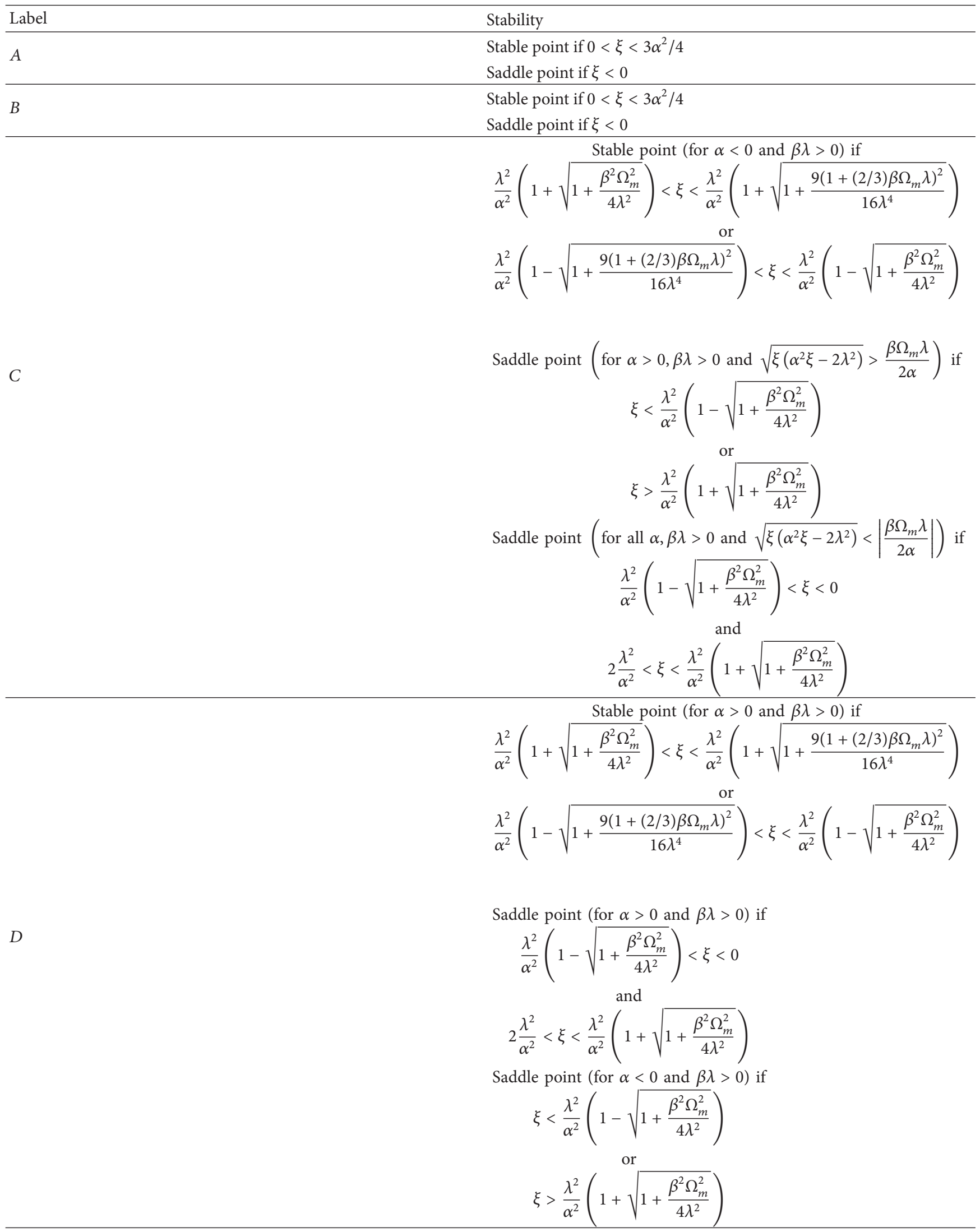




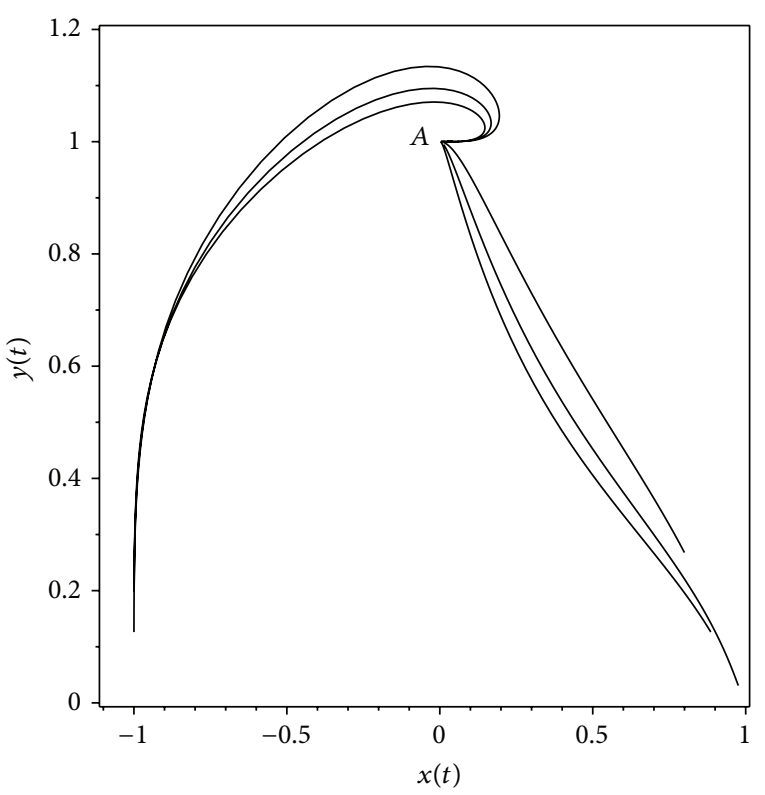

(a)

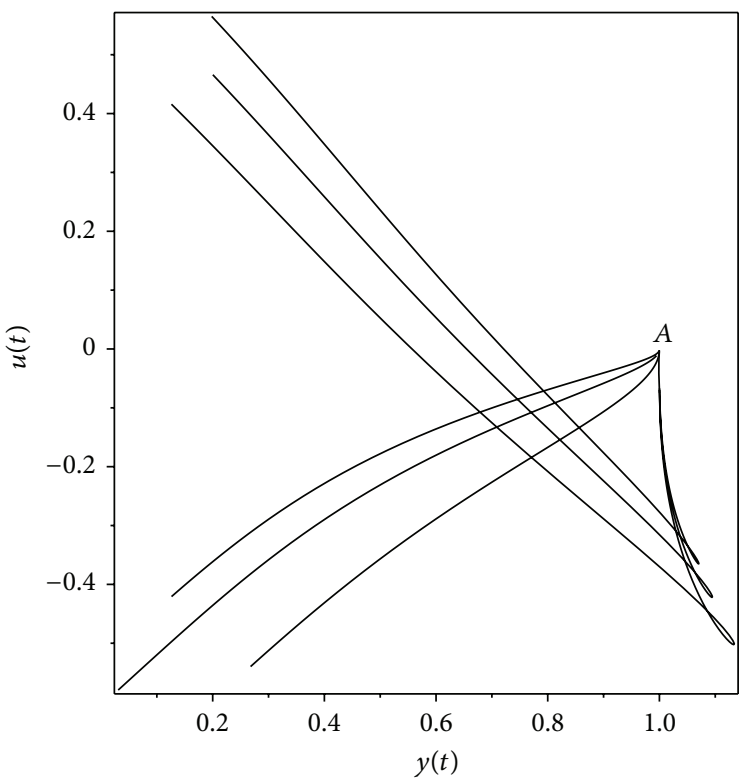

(b)

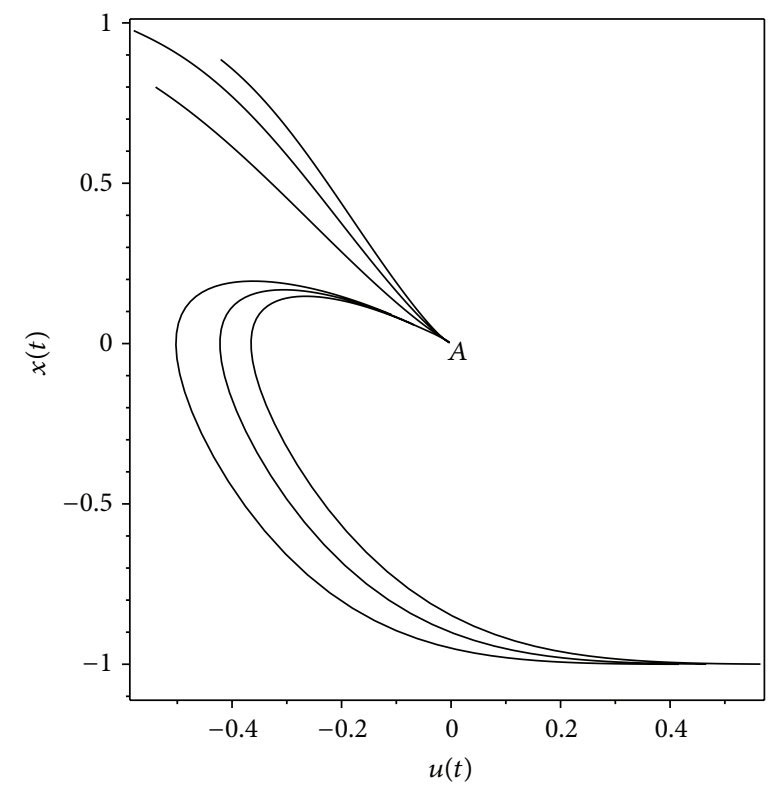

(c)

Figure 1: From (a) to (c), the projections of the phase-space trajectories on the $y-x, u-y$, and $x-u$ planes with $\xi=0.5$ and $\alpha=1.5$. For these values of the parameters, point $A$ is a stable attractor.

or

$$
\begin{array}{r}
\frac{\lambda^{2}}{\alpha^{2}}\left(1-\sqrt{1+\frac{9\left(1+(2 / 3) \beta \Omega_{m} \lambda\right)^{2}}{16 \lambda^{4}}}\right) \\
<\xi<\frac{\lambda^{2}}{\alpha^{2}}\left(1-\sqrt{1+\frac{\beta^{2} \Omega_{m}^{2}}{4 \lambda^{2}}}\right) .
\end{array}
$$

Universe at this point behaves like a cosmological constant with $\omega_{\mathrm{DE}}=-1$. In Figure 2, where the chosen values of the parameters $(\xi=0.92$ and $\alpha=-1.5)$ satisfy existing condition and constraints (22) or (23), the projections of the phasespace trajectories on $y-x, u-y$, and $x-u$ are plotted, respectively. Three-dimensional plot is also shown in Figure 4. Point $C$ could be a saddle point if the constraints (22) or (23) are not satisfied as it is mentioned in Table 3.

Critical Point D. Similar to the critical point $C$, the fixed point $D$ could be an attractor of the model. In this situation the parameter $\xi$ should be satisfied again in constraint (22) or (23). The only difference is that the point $D$ is stable for 


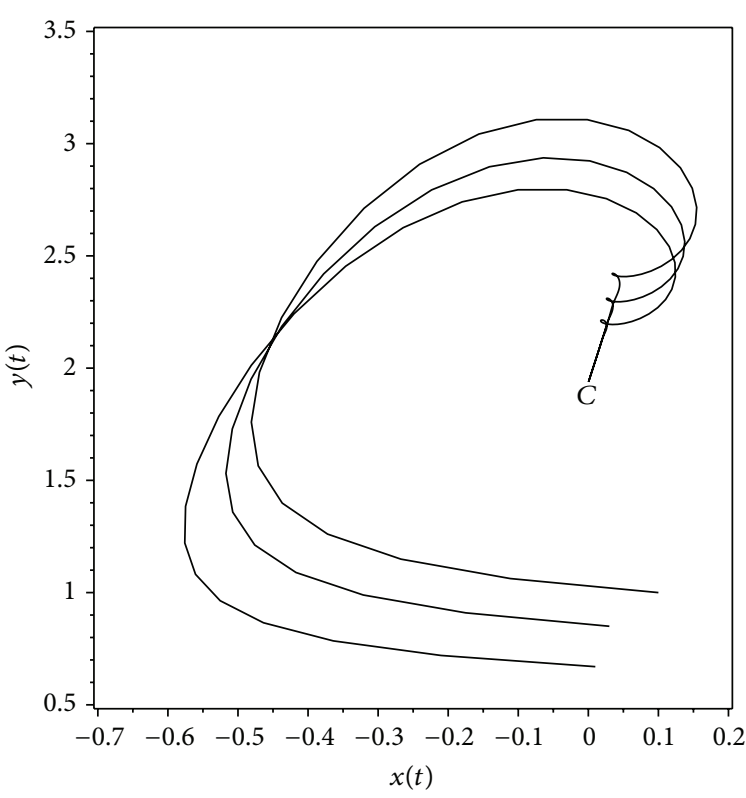

(a)

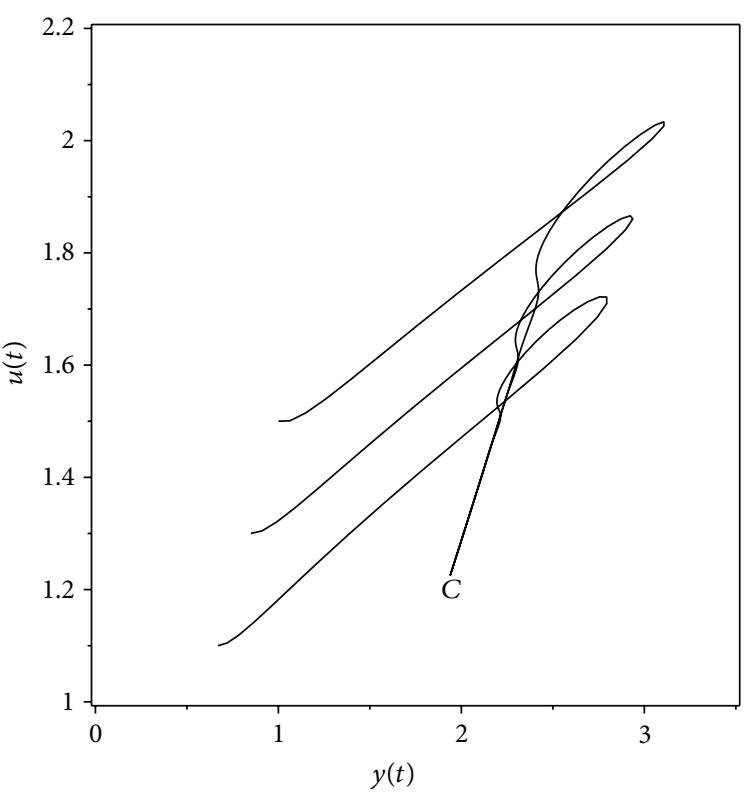

(b)

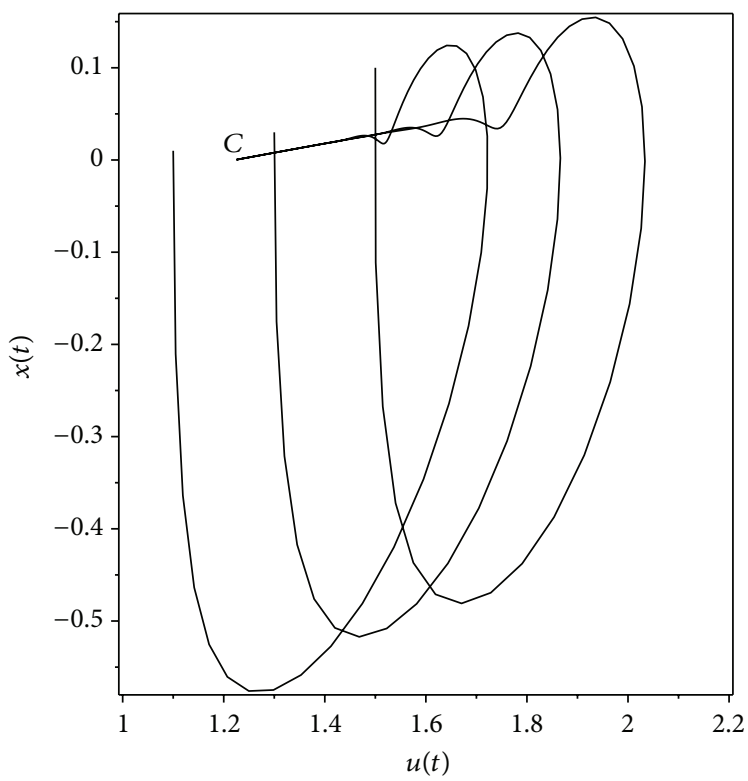

(c)

Figure 2: From (a) to (c), the projections of the phase-space trajectories on the $y-x, u-y$, and $x-u$ planes with $\xi=0.92$ and $\alpha=-1.5$. The trajectories are attracted by the point $C$.

positive values of $\alpha$ while $C$ is stable for negative values of $\alpha$. In Figure 3 we have chosen the parameters such that the fixed point $D$ is an attractor point of the model $(\xi=0.92$ and $\alpha=$ 1.5) and Figure 4 shows the corresponding 3-dimensional plot. Conditions needed in order for $D$ to become a saddle point are presented in Table 3.

\section{Conclusion}

Tachyonic teleparallel dark energy is a generalization of the teleparallel dark energy recently proposed by Geng et al.
[84]. Such an extension is obtained by replacing a canonical scalar field (quintessence) by a noncanonical scalar field (tachyon). Dynamics of tachyonic teleparallel dark energy has been studied in [90]. In this paper we performed a detailed phase-space analysis of interacting tachyonic teleparallel dark energy where the dark energy sector interacts with the dark matter one. We extracted the critical points of the model and examined their stabilities. We derived the eigenvalues corresponding to each critical point in order to find the stable solutions. We also calculated some of the important cosmological parameters such as the equation of state of dark 


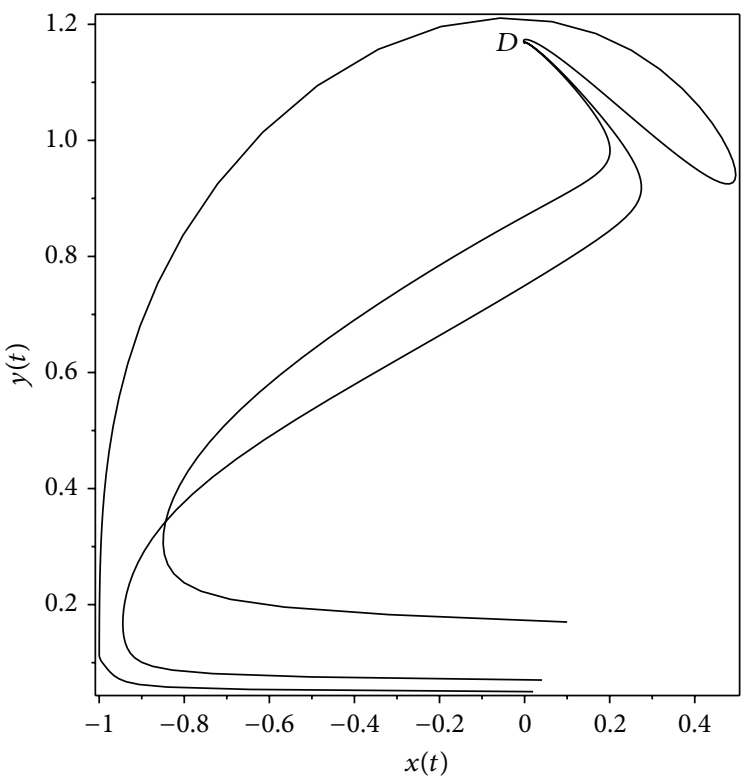

(a)

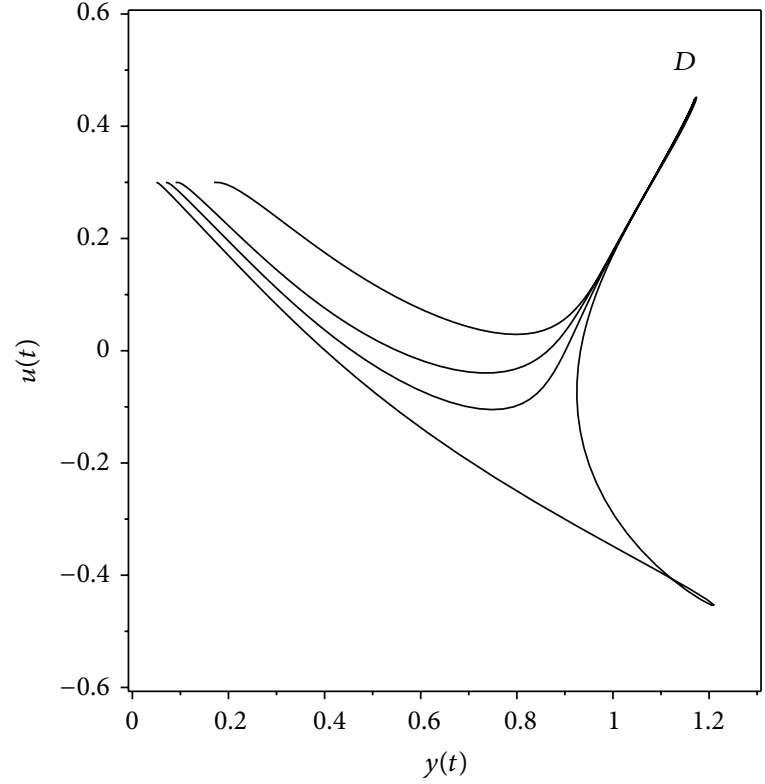

(b)

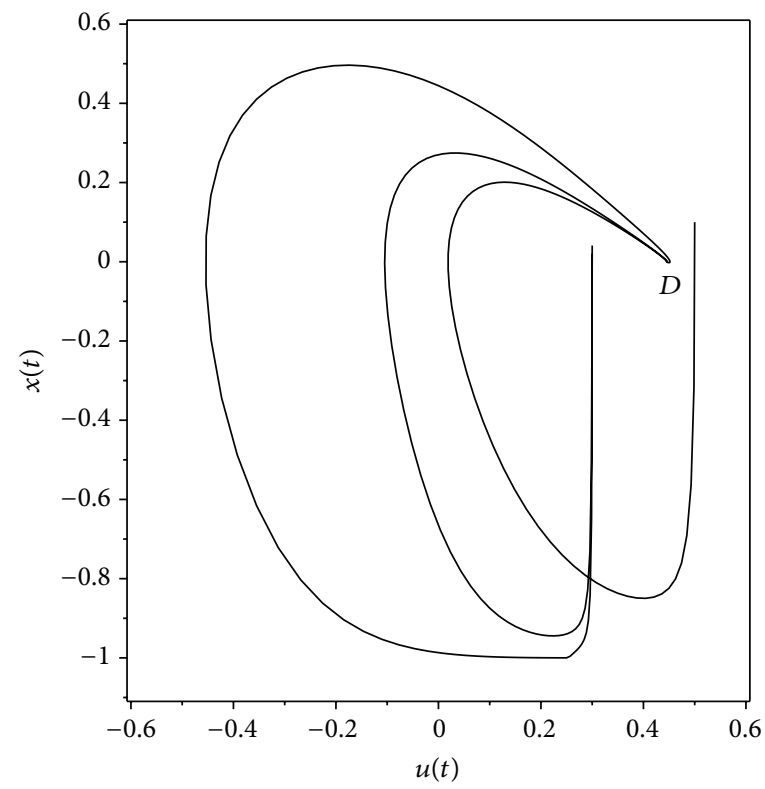

(c)

Figure 3: From (a) to (c), the projections of the phase-space trajectories on the $y-x, u-y$, and $x-u$ planes with $\xi=0.92$ and $\alpha=1.5$. The trajectories are attracted by the point $D$.

energy $\omega_{\mathrm{DE}}$, the total equation of state $\omega_{\text {tot }}$, and deceleration parameter $q$ in order to find whether critical points correspond to an accelerating universe or not. Depending on the nonminimal coupling parameter $\xi$, we found three stable attractors of the model, that is, the point $A$ for a constant potential and points $C$ and $D$ if $\xi$ satisfies (22) or (23). In order to show behavior of the model at the critical points more transparently, we plotted two- and three-dimensional phase-space trajectories of the model in Figures 1-4. These figures show that by choosing parameters of the model suitably, points $A, C$, and $D$ are attractors of the model. While all of the stable solutions admit an accelerating universe, they correspond to a complete dark energy domination and thus they are unable to solve coincidence problem. One can consider more complicated interaction terms constructed to solve the coincidence problem [117] or generalize the nonminimal coupling function in order to have a varying parameter $\alpha$ similar to the procedure which was done in 


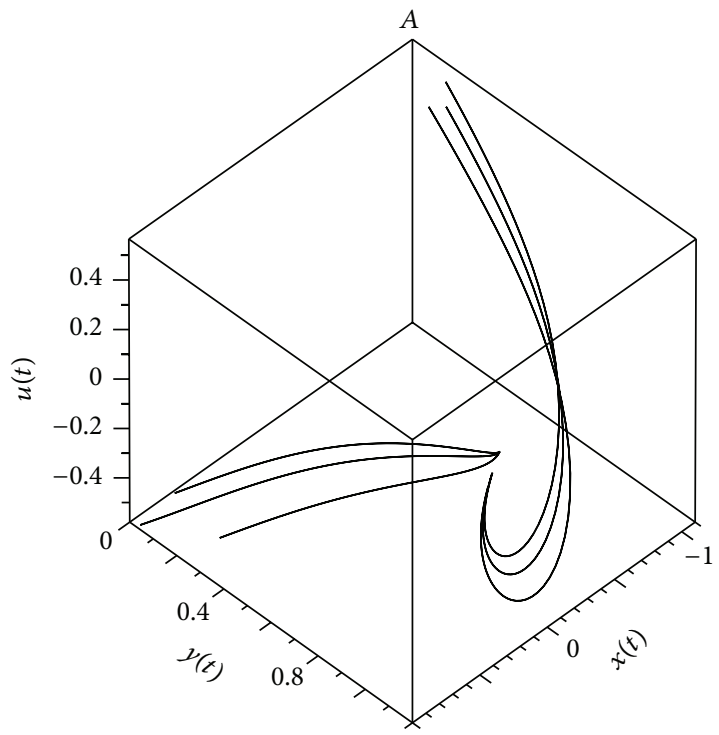

(a)

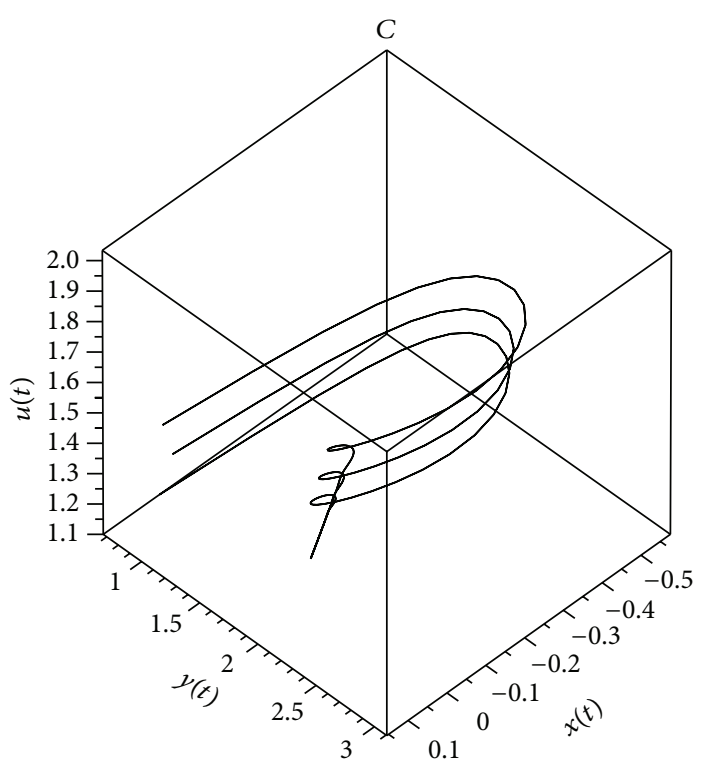

(b)

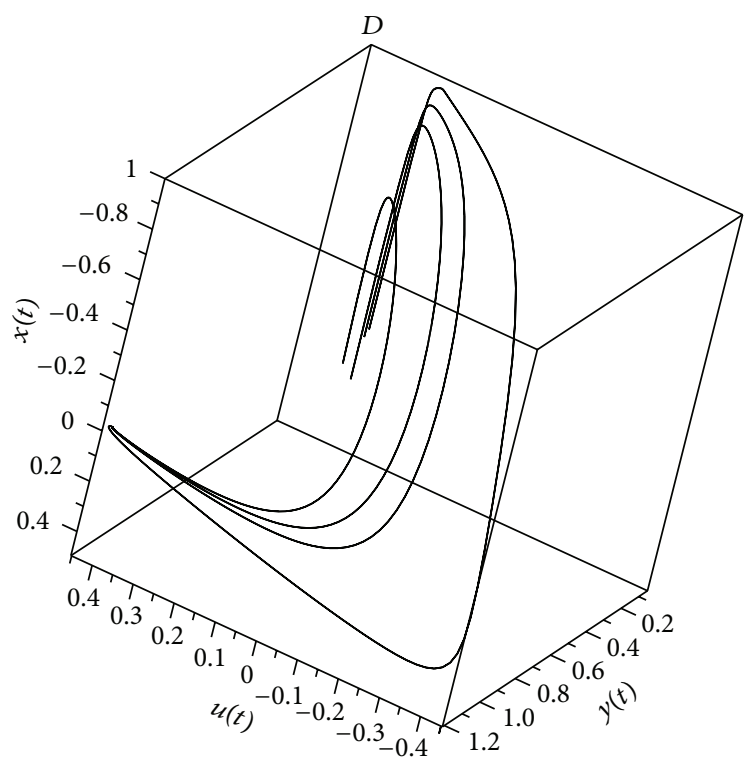

(c)

FIgURE 4: Three-dimensional phase-space trajectories of the model with stable attractors $A(\mathrm{a}), C(\mathrm{~b})$, and $D$ (c), respectively.

[88]. Moreover, in order to show that there are no significant equilibrium points lost at infinity one can use the Poincare central projection method to investigate the dynamics at infinity [118]. Such method has been applied for teleparallel dark energy model in [86].

\section{Appendix}

\section{Stability of the Critical Points}

The elements of $3 \times 3$ matrix $M$ of the linearized perturbation equations for the real and physically meaningful critical points $\left(x_{c}, y_{c}, u_{c}\right)$ of the autonomous system read

$$
\begin{aligned}
M_{11}= & \sqrt{3}\left(-2 \lambda x_{c} y_{c}-\sqrt{3}\left(1-3 x_{c}^{2}\right)\right. \\
& \left.+6 \alpha \xi u_{c} x_{c} \mu_{c}^{-1} y_{c}^{-1}\right), \\
M_{12}= & \sqrt{3} \mu_{c}^{-2}\left(\lambda+2 \alpha \xi u_{c} \mu_{c}^{-1} y_{c}^{-2}\right)+\sqrt{3} \beta y_{c}^{-2} \Omega_{m}, \\
M_{13}= & -2 \sqrt{3} \alpha \xi \mu_{c}^{-3} y_{c}^{-1} \\
M_{21}= & \frac{y_{c}^{2}\left(-3 \mu_{c}^{3} x_{c} y_{c}\left(x_{c}^{2}+\gamma-2\right)+4 \sqrt{3} \alpha \xi u_{c}\right)}{2\left(2 \xi u_{c}^{2}+1\right)} \\
& -\frac{\sqrt{3} \lambda y_{c}^{2}}{2},
\end{aligned}
$$




$$
\begin{aligned}
M_{22}= & \frac{y_{c}\left(9 \mu_{c} y_{c}\left(x_{c}^{2}-\gamma\right)+8 \sqrt{3} \alpha \xi x_{c} u_{c}\right)}{2\left(2 \xi u_{c}^{2}+1\right)} \\
& -\sqrt{3} \lambda x_{c} y_{c}+\frac{3 \gamma}{2} \\
M_{23}= & \frac{2 \sqrt{3} \xi y_{c}^{2}\left(-\sqrt{3} \mu_{c} u_{c} y_{c}\left(x_{c}^{2}-\gamma\right)+2 \alpha x_{c}\right)}{\left(2 \xi u_{c}^{2}+1\right)^{2}} \\
& -\frac{2 \sqrt{3} \alpha \xi x_{c} y_{c}^{2}}{2 \xi u_{c}^{2}+1}, \\
M_{31}= & \frac{\sqrt{3} \alpha y_{c}}{2} \\
M_{32}= & \frac{\sqrt{3} \alpha x_{c}}{2} \\
M_{33}= & 0 .
\end{aligned}
$$

Examining the eigenvalues of the matrix $M$ for each critical point, one determines its stability behavior. We mentioned that although the matrix $M$ has a complicated form, inserting the explicit critical points presented in Table 1 into the elements, it takes a simple form and we can easily calculate its eigenvalues. The corresponding eigenvalues for each critical point are presented in Table 2.

\section{Conflict of Interests}

The author declares that there is no conflict of interests regarding the publication of this paper.

\section{References}

[1] S. Perlmutter, G. Aldering, G. Goldhaber et al., "Measurements of $\Omega$ and $\Lambda$ from 42 high-redshift supernovae," The Astrophysical Journal, vol. 517, no. 2, p. 565, 1999.

[2] A. G. Riess, A. V. Filippenko, P. Challis et al., "Observational evidence from supernovae for an accelerating universe and a cosmological constant," The Astronomical Journal, vol. 116, no. 3, p. 1009, 1998.

[3] U. Seljak, A. Makarov, P. McDonald et al., "Cosmological parameter analysis including SDSS Ly $\alpha$ forest and galaxy bias: constraints on the primordial spectrum of fluctuations, neutrino mass, and dark energy," Physical Review D, vol. 71, Article ID 103515, 2005.

[4] M. Tegmark, M. Strauss, M. Blanton et al., "Cosmological parameters from SDSS and WMAP," Physical Review D, vol. 69, Article ID 103501, 2004.

[5] D. J. Eisenstein, I. Zehavi, D. W. Hogg et al., "Detection of the baryon acoustic peak in the large-scale correlation function of SDSS ILuminous red galaxies," The Astrophysical Journal, vol. 633 , no. 2, p. 560, 2005.

[6] D. N. Spergel, L. Verde, H. V. Peiris et al., "First-year Wilkinson Microwave Anisotropy probe (WMAP) observations: determination of cosmological parameters," The Astrophysical Journal Supplement Series, vol. 148, no. 1, p. 175, 2003.

[7] E. Komatsu et al., WMAP Collaboration.
[8] G. Hinshaw, D. Larson, E. Komatsu et al., "Nine-year Wilkinson Microwave Anisotropy Probe (WMAP) observations: cosmological parameter results," The Astrophysical Journal Supplement Series, vol. 208, no. 2, p. 19, 2013.

[9] P. A. R. Ade, N. Aghanim, and C. Armitage-Caplan, "Planck 2013 results. XVI. Cosmological parameters," Astronomy \& Astrophysics, 2013.

[10] S. Nojiri and S. D. Odintsov, "Unified cosmic history in modified gravity: from $f(R)$ theory to Lorentz non-invariant models," Physics Reports, vol. 505, no. 2-4, pp. 59-144, 2011.

[11] S. Capozziello and V. Faraoni, Beyond Einstein Gravity, Springer, 2010.

[12] S. Capozziello and M. De Laurentis, "Extended theories of gravity," Physics Reports, vol. 509, no. 4-5, pp. 167-321, 2011.

[13] A. de la Cruz-Dombriz and D. Saez-Gomez, "Black holes, cosmological solutions, future singularities, and their thermodynamical properties in modified gravity theories," Entropy, vol. 14, no. 9, pp. 1717-1770, 2012.

[14] T. Padmanabhan, "Cosmological constant-the weight of the vacuum," Physics Reports, vol. 380, pp. 235-320, 2003.

[15] S. M. Carroll, "The cosmological constant," Living Reviews in Relativity, vol. 4, no. 1, 2001.

[16] V. Sahni and A. Starobinsky, "The case for a positive cosmological lambda term," International Journal of Modern Physics D, vol. 9, pp. 373-444, 2000.

[17] P. J. E. Peebles and B. Ratra, "The cosmological constant and dark energy," Reviews of Modern Physics, vol. 75, p. 559, 2003.

[18] B. Ratra and P. J. E. Peebles, "Cosmological consequences of a rolling homogeneous scalar field," Physical Review D, vol. 37, p. 3406,1988 .

[19] C. Wetterich, "Cosmology and the fate of dilatation symmetry," Nuclear Physics B, vol. 302, no. 4, pp. 668-696, 1988.

[20] A. R. Liddle and R. J. Scherrer, "Classification of scalar field potentials with cosmological scaling solutions," Physical Review $D$, vol. 59, Article ID 023509, 1998.

[21] I. Zlatev, L. M. Wang, and P. J. Steinhardt, "Quintessence, cosmic coincidence, and the cosmological constant," Physical Review Letters, vol. 82, p. 896, 1999.

[22] Z. K. Guo, N. Ohta, and Y. Z. Zhang, "Parametrizations of the dark energy density and scalar potentials," Modern Physics Letters A, vol. 22, no. 12, p. 883, 2007.

[23] C. Armendariz-Picon, V. F. Mukhanov, and P. J. Steinhardt, "Essentials of $k$-essence," Physical Review D, vol. 63, Article ID 103510, 2001.

[24] R. R. Caldwell, "A phantom menace? Cosmological consequences of a dark energy component with super-negative equation of state," Physics Letters B, vol. 545, no. 1-2, pp. 23-29, 2002.

[25] S. Nojiri and S. D. Odintsov, "Quantum de sitter cosmology and phantom matter," Physics Letters B, vol. 562, no. 3-4, p. 147, 2003.

[26] V. K. Onemli and R. P. Woodard, "Quantum effects can render $w<-1$ on cosmological scales," Physical Review D, vol. 70, Article ID 107301, 2004.

[27] M. R. Setare, "Interacting holographic phantom," High Energy Physics, vol. 50, pp. 991-998, 2007.

[28] E. N. Saridakis, "Theoretical limits on the equation-of-state parameter of phantom cosmology," Physics Letters B, vol. 676, pp. 7-11, 2009.

[29] R. R. Caldwell, M. Kamionkowski, and N. N. Weinberg, "Phantom energy: dark energy with $w<-1$ causes a cosmic 
doomsday," Physical Review Letters, vol. 91, Article ID 071301, 2003.

[30] A. Sen, “Tachyon matter," Journal of High Energy Physics, vol. 207, p. 65, 2002.

[31] A. Sen, "Field theory of tachyon matter," Modern Physics Letters $A$, vol. 17, no. 27, p. 1797, 2002.

[32] B. Feng, X. L. Wang, and X. M. Zhang, "Dark energy constraints from the cosmic age and supernova," Physics Letters B, vol. 607, no. 1-2, pp. 35-41, 2005.

[33] Z. K. Guo, Y. S. Piaoc, X. Zhang et al., "Cosmological evolution of a quintom model of dark energy," Physics Letters B, vol. 608, no. 3-4, pp. 177-182, 2005.

[34] M. Li, B. Feng, and X. Zhang, "A single scalar field model of dark energy with equation of state crossing-1," Journal of Cosmology and Astroparticle Physics, vol. 512, no. 2, 2005.

[35] B. Feng, M. Li, Y.-S. Piao, and X. Zhang, "Oscillating quintom and the recurrent universe," Physics Letters B, vol. 634, no. 2-3, pp. 101-105, 2006.

[36] M. R. Setare, "Generalized second law of thermodynamics in quintom dominated universe," Physics Letters B, vol. 641, no. 2, pp. 130-133, 2006.

[37] W. Zhao and Y. Zhang, "Quintom models with an equation of state crossing -1," Physical Review D, vol. 73, Article ID 123509, 2006.

[38] M. R. Setare and E. N. Saridakis, "Non-minimally coupled canonical, phantom and quintom models of holographic dark energy," Physics Letters B, vol. 671, no. 3, pp. 331-338, 2009.

[39] Y.-F. Cai, E. N. Saridakis, M. R. Setare, and J.-Q. Xia, "Quintom cosmology: theoretical implications and observations," Physics Reports, vol. 493, no. 1, pp. 1-60, 2010.

[40] K. Bamba, S. Capozziello, S. Nojiri, and S. D. Odintsov, "Dark energy cosmology: the equivalent description via different theoretical models and cosmography tests," Astrophysics and Space Science, vol. 342, no. 1, pp. 155-228, 2012.

[41] B. L. Spokoiny, "Inflation and generation of perturbations in broken-symmetric theory of gravity," Physics Letters B, vol. 147, no. 1-3, pp. 39-43, 1984.

[42] F. Perrotta, C. Baccigalupi, and S. Matarrese, "Extended quintessence," Physical Review D, vol. 61, Article ID 023507, 1999.

[43] V. Faraoni, "Inflation and quintessence with nonminimal coupling," Physical Review D, vol. 62, Article ID 023504, 2000.

[44] E. Elizalde, S. Nojiri, and S. D. Odintsov, "Late-time cosmology in a (phantom) scalar-tensor theory: dark energy and the cosmic speed-up," Physical Review D, vol. 70, Article ID 043539, 2004.

[45] O. Hrycyna and M. Szydlowski, "Non-minimally coupled scalar field cosmology on the phase plane," Journal of Cosmology and Astroparticle Physics, vol. 2009, p. 26, 2009.

[46] O. Hrycyna and M. Szydlowski, "Extended quintessence with nonminimally coupled phantom scalar field," Physical Review $D$, vol. 76, Article ID 123510, 2007.

[47] R. C. de Souza and G. M. Kremer, "Constraining non-minimally coupled tachyon fields by the Noether symmetry," Classical and Quantum Gravity, vol. 26, no. 13, Article ID 135008, 2009.

[48] A. A. Sen and N. C. Devi, "Cosmology with non-minimally coupled $k$-field," General Relativity and Gravitation, vol. 42, no. 4, pp. 821-838, 2010.

[49] L. Amendola, “Coupled quintessence," Physical Review D, vol. 62, Article ID 043511, 2000.
[50] B. Gumjudpai, T. Naskar, M. Sami, and S. Tsujikawa, "Coupled dark energy: towards a general description of the dynamics," Journal of Cosmology and Astroparticle Physics, vol. 506, p. 7, 2005.

[51] A. P. Billyard and A. A. Coley, "Interactions in scalar field cosmology," Physical Review D, vol. 61, Article ID 083503, 2000.

[52] J. P. Mimoso, A. Nunes, and D. Pavon, "Asymptotic behavior of the warm inflation scenario with viscous pressure," Physical Review D, vol. 73, Article ID 023502, 2006.

[53] R. Lazkoz and G. Leon, "Quintom cosmologies admitting either tracking or phantom attractors," Physics Letters B, vol. 638, no. 4, pp. 303-309, 2006.

[54] T. Gonzalez, G. Leon, and I. Quiros, "Dynamics of quintessence models of dark energy with exponential coupling to dark matter," Classical and Quantum Gravity, vol. 23, no. 9, p. 3165, 2006.

[55] G. R. Farrar and P. J. E. Peebles, "Interacting dark matter and dark energy," The Astrophysical Journal, vol. 604, no. 1, 2004.

[56] B. Wang, Y. G. Gong, and E. Abdalla, "Transition of the dark energy equation of state in an interacting holographic dark energy model," Physics Letters B, vol. 624, no. 3-4, pp. 141-1446, 2005.

[57] M. R. Setare, "Interacting holographic dark energy model in non-flat universe," Physics Letters B, vol. 642, no. 1-2, pp. 1-4, 2006.

[58] Z. K. Guo, R. G. Cai, and Y. Z. Zhang, "Cosmological evolution of interacting phantom energy with dark matter," Journal of Cosmology and Astroparticle Physics, vol. 505, p. 2, 2005.

[59] A. Nunes, J. P. Mimoso, and T. C. Charters, "Scaling solutions from interacting fluids," Physical Review D, vol. 63, Article ID 083506, 2001.

[60] D. F. Mota and C. van de Bruck, "On the spherical collapse model in dark energy cosmologies," Astronomy \& Astrophysics, vol. 421, no. 1, pp. 71-81, 2004.

[61] M. Manera and D. F. Mota, "Cluster number counts dependence on dark energy inhomogeneities and coupling to dark matter," Mathematics \& Physical Sciences, vol. 371, no. 3, pp. 1373-1380, 2006.

[62] N. J. Nunes and D. F. Mota, "Structure formation in inhomogeneous dark energy models," Mathematics \& Physical Sciences, vol. 368, no. 2, pp. 751-758, 2006.

[63] J. D. Barrow and T. Clifton, "Cosmologies with energy exchange," Physical Review D, vol. 73, Article ID 103520, 12 pages, 2006.

[64] T. Clifton and J. D. Barrow, "Decaying gravity," Physical Review $D$, vol. 73, Article ID 104022, 2006.

[65] T. Clifton and J. D. Barrow, "Ups and downs of cyclic universes," Physical Review D, vol. 75, Article ID 043515.

[66] A. Einstein, "Translation of Einstein's attempt of a unified field theory with teleparallelism," Sitzungsberichte der Preussischen Akademie der Wissenschaften, p. 217, 1982, ibid p. 224; A. Unzicker and T. Case.

[67] K. Hayashi and T. Shirafuji, "New general relativity," Physical Review D, vol. 19, p. 3524, 1979.

[68] K. Hayashi and T. Shirafuji, "New general relativity," Physical Review D, vol. 24, pp. 3312-3314, 1982.

[69] G. R. Bengochea and R. Ferraro, "Dark torsion as the cosmic speed-up," Physical Review D, vol. 79, Article ID 124019, 2009.

[70] E. V. Linder, "Einstein's other gravity and the acceleration of the Universe," Physical Review D, vol. 81, Article ID 127301, 2010. 
[71] E. V. Linder, "Erratum: Einstein's other gravity and the acceleration of the Universe [Phys. Rev. D 81, 127301 (2010)]," Physical Review D, vol. 82, Article ID 109902, 2010.

[72] H. Wei, X. P. Ma, and H. Y. Qi, " $f(T)$ theories and varying fine structure constant," Physics Letters B, vol. 703, no. 1, pp. 74-80, 2011.

[73] P. X. Wu and H. W. Yu, “ $f(T)$ models with phantom divide line crossing," The European Physical Journal C, vol. 71, p. 1552, 2011.

[74] P. X. Wu and H. W. Yu, "The stability of the Einstein static state in $f(T)$ gravity," Physics Letters B, vol. 703, no. 3, pp. 223-227, 2011.

[75] K. Bamba, C. Q. Geng, C. C. Lee, and L. W. Luo, "Equation of state for dark energy in $f(T)$ gravity," Joint Center for Artificial Photosynthesis, vol. 1101, no. 021, 2011.

[76] K. Bamba, C. Q. Geng, and C. C. Lee, "Comment on 'Einstein's OtherGravity and the Acceleration of the Universe," http://xxx.lanl.gov/abs/1008.4036.

[77] K. Bamba and C. Q. Geng, "Thermodynamics of cosmological horizons in $f(T)$ gravity," Journal of Cosmology and Astroparticle Physics, vol. 1111, p. 008, 2011.

[78] Y. Zhang, H. Li, Y. G. Gong, and Z. H. Zhu, "Notes on $f(T)$ theories," Journal of Cosmology and Astroparticle Physics, vol. 1107, p. 015, 2011.

[79] R. Ferraro and F. Fiorini, "Spherically symmetric static spacetimes in vacuum $f(T)$ gravity," Physical Review D, vol. 84, Article ID 083518.

[80] M. Sharif and S. Rani, " $f(T)$ models within bianchi type-i universe," Modern Physics Letters A, vol. 26, no. 22, p. 1657, 2011.

[81] R. Myrzakulov, "Accelerating universe from $f(T)$ gravity," The European Physical Journal C, vol. 71, p. 1752, 2011.

[82] B. Li, T. P. Sotiriou, and J. D. Barrow, “ $f(T)$ gravity and local Lorentz invariance," Physical Review D, vol. 83, Article ID 064035, 2011.

[83] T. P. Sotiriou, B. Li, and J. D. Barrow, "Generalizations of teleparallel gravity and local Lorentz symmetry," Physical Review D, vol. 83, Article ID 104030.

[84] C.-Q. Geng, C.-C. Lee, E. N. Saridakis, and Y.-P. Wu, “Teleparallel' dark energy," Physics Letters B, vol. 704, no. 5, pp. 384-387, 2011.

[85] C.-Q. Geng, C.-C. Lee, and E. N. Saridakis, "Observational constraints on teleparallel dark energy," Journal of Cosmology and Astroparticle Physics, vol. 1201, p. 2, 2012.

[86] C. Xu, E. N. Saridakis, and G. Leon, "Phase-space analysis of teleparallel dark energy," Journal of Cosmology and Astroparticle Physics, vol. 1207, 23 pages, 2012.

[87] H. Wei, "Dynamics of teleparallel dark energy," Physics Letters $B$, vol. 712, no. 4-5, pp. 430-436, 2012.

[88] G. Otalora, "Scaling attractors in interacting teleparallel dark energy," Journal of Cosmology and Astroparticle Physics, vol. 1307, p. 044, 2013.

[89] A. Banijamali and B. Fazlpour, "Tachyonic teleparallel dark energy," Astrophysics and Space Science, vol. 342, no. 1, pp. 229235, 2012.

[90] G. Otalora, "Cosmological dynamics of tachyonic teleparallel dark energy," Physical Review D, vol. 88, Article ID 063505, 2013.

[91] R. Weitzenböck, Invarianten Theorie, Nordho, Groningen, The Netherlands, 1923.

[92] K. Hayashi and T. Shirafuji, "New general relativity," Physical Review D, vol. 19, p. 3524, 1979.
[93] J. M. Aguirregabiria and R. Lazkoz, "Tracking solutions in tachyon cosmology," Physical Review D, vol. 69, Article ID 123502, 2004.

[94] E. J. Copeland, M. R. Garousi, M. Sami, and S. Tsujikawa, "What is needed of a tachyon if it is to be the dark energy?" Physical Review D, vol. 71, Article ID 043003, 2005.

[95] F. Huang, K. Xiao, and J. -Y. Zhu, "the dynamics of tachyon field with an inverse square potential in loop quantum cosmology," International Journal of Modern Physics D, vol. 22, no. 6, Article ID 1350030, 15 pages, 2013.

[96] I. Quiros, T. Gonzalez, D. Gonzalez, Y. Napoles, R. GarciaSalcedo, and C. Moreno, "Study of tachyon dynamics for broad classes of potentials," Classical and Quantum Gravity, vol. 27, Article ID 215021, 13 pages, 2010.

[97] E. J. Copeland, A. R. Liddle, and D. Wands, "Exponential potentials and cosmological scaling solutions," Physical Review D, vol. 57, p. 4686, 1998.

[98] P. G. Ferreira and M. Joyce, "Structure formation with a selftuning scalar field," Physical Review Letters, vol. 79, p. 4740, 1997.

[99] Y. Gong, A. Wang, and Y.-Z. Zhang, "Exact scaling solutions and fixed points for general scalar field," Physics Letters B, vol. 636, no. 5, pp. 286-292, 2006.

[100] X.-M. Chen and Y. Gong, "Fixed points in interacting dark energy models," Physics Letters B, vol. 675, no. 1, pp. 9-13, 2009.

[101] A. A. Coley, J. Wainwright, and G. F. R. Ellis, Dynamical Systems in Cosmology, Cambridge University Press, Cambridge, Mass, USA, 1997.

[102] A. A. Coley, Astrophysics and Space Science Library, Springer, 2004.

[103] E. J. Copeland, A. R. Liddle, and D. Wands, "Exponential potentials and cosmological scaling solutions," Physical Review D, vol. 57, p. 4686, 1998.

[104] L. Amendola, "Scaling solutions in general nonminimal coupling theories," Physical Review D, vol. 60, Article ID 043501, 1999.

[105] R. G. Cai and A. Wang, "Cosmology with interaction between phantom dark energy and dark matter and the coincidence problem," Journal of Cosmology and Astroparticle Physics, vol. 503, p. 2, 2005.

[106] H. Wei and R. G. Cai, " $K$-chameleon and the coincidence problem," Physical Review D, vol. 71, Article ID 043504, 2005.

[107] E. J. Copeland, M. Sami, and S. Tsujikawa, "Dynamics of dark energy," International Journal of Modern Physics D, vol. 15, no. 11, p. 1753, 2006.

[108] A. De Felice and S. Tsujikawa, “ $f(R)$ Theories," Living Reviews in Relativity, vol. 13, p. 3, 2010.

[109] T. P. Sotiriou and V. Faraoni, " $f(R)$ theories of gravity," Reviews of Modern Physics, vol. 82, p. 451, 2010.

[110] S. Tsujikawa, "Modified gravity models of dark energy," Lectures on Cosmology, vol. 800, pp. 99-145, 2010.

[111] R. J. Scherrer and A. Sen, "Phantom dark energy models with a nearly flat potential," Physical Review D, vol. 78, Article ID 067303, 2008.

[112] M. Setare and E. N. Saridakis, "Quintom dark energy models with nearly flat potentials," Physical Review D, vol. 79, Article ID 043005, 2009.

[113] R. Lazkoz and G. Leon, "Quintom cosmologies admitting either tracking or phantom attractors," Physics Letters B, vol. 638, no. 4, pp. 303-309, 2006. 
[114] T. Gonzalez, G. Leon, and I. Quiros, "Dynamics of quintessence models of dark energy with exponential coupling to the dark matter," Classical and Quantum Gravity, vol. 23, p. 3165, 2006.

[115] T. Clifton and J. D. Barrow, "Decaying gravity," Physical Review D, vol. 73, Article ID 104022, 2006.

[116] B. Gumjudpai, T. Naskar, M. Sami, and S. Tsujikawa, "Coupled dark energy: towards a general description of the dynamics," Journal of Cosmology and Astroparticle Physics, vol. 506, p. 7, 2005.

[117] X.-M. Chen, Y. Gong, and E. N. Saridakis, "Phase-space analysis of interacting phantom cosmology," Joint Center for Artificial Photosynthesis, vol. 904, p. 1, 2009.

[118] S. Lynch, Dynamical Systems With Applications Using Mathematica, Birkhauser, Boston, Mass, USA, 2007. 

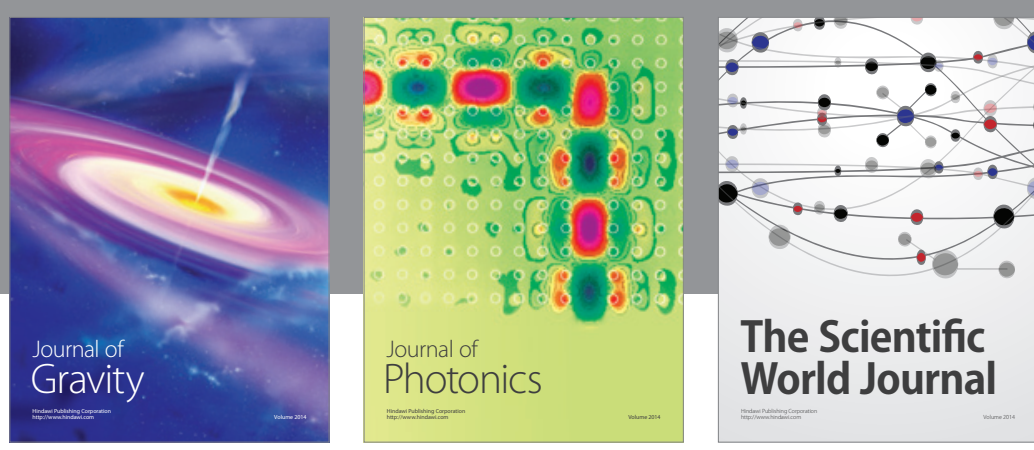

The Scientific World Journal
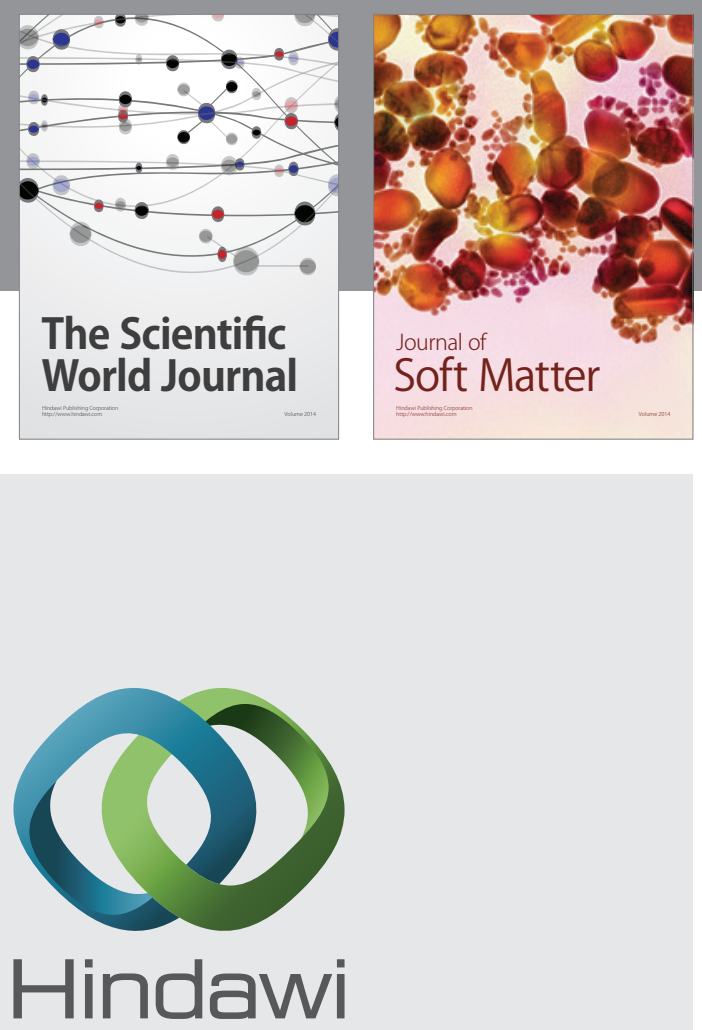

Submit your manuscripts at

http://www.hindawi.com

nternational Journal of

Statistical Mechanics
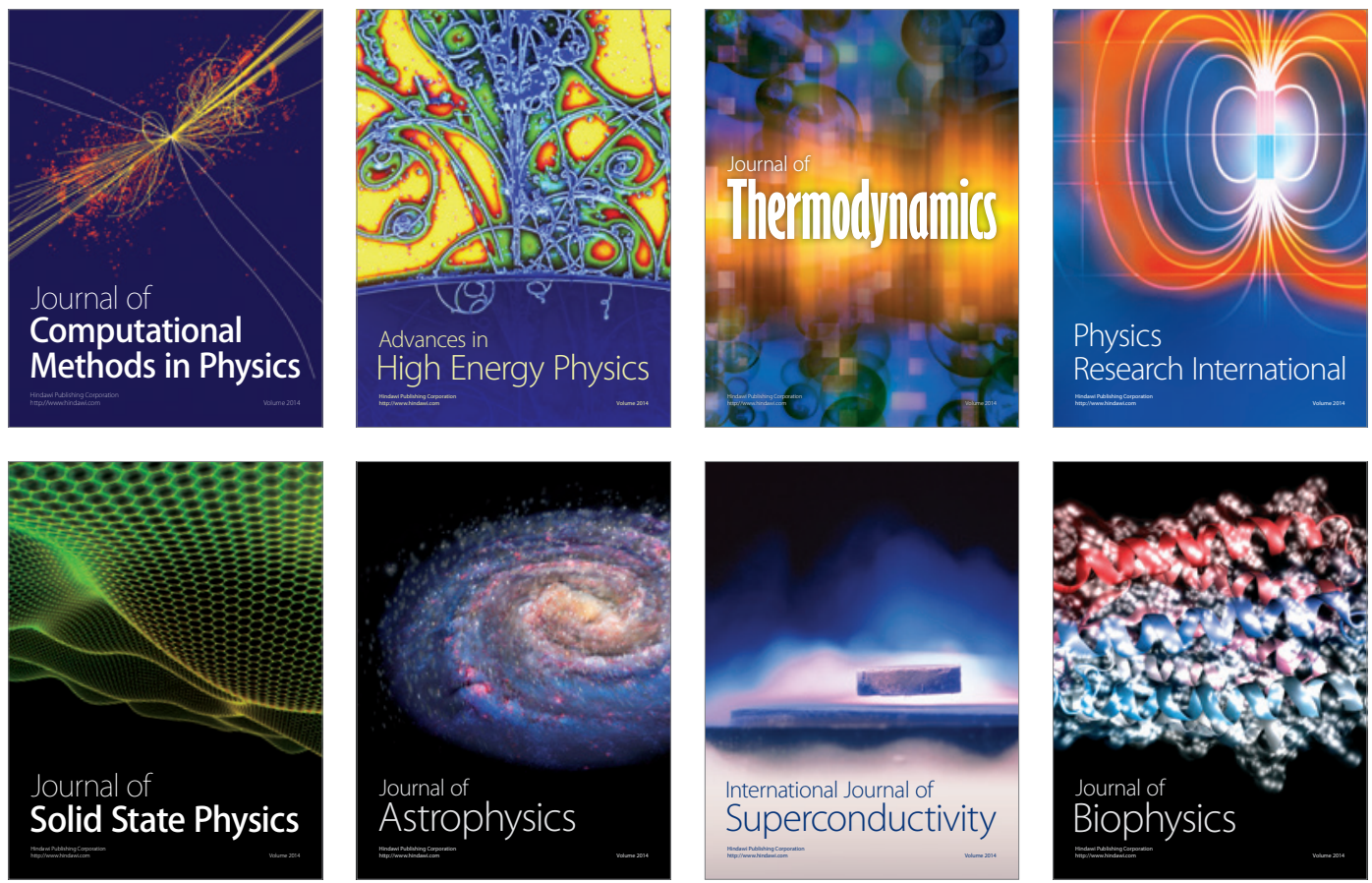
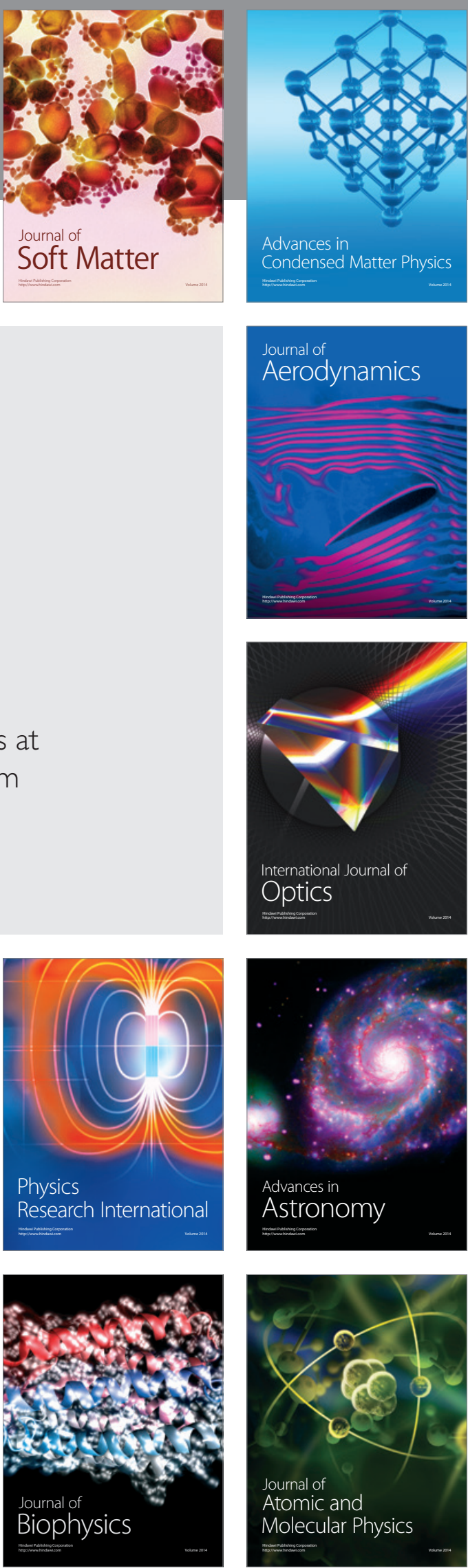